\title{
TRATAMENTO DAS COMPLICAÇÕES NEUROLÓGICAS DA VACINAÇÃO ANTI-RÁBICA
}

\author{
Paulo A. P. Saraiva* \\ J. LAMARTine DE Assis **
}

No Estado de São Paulo ainda é freqüente a necessidade de vacinação anti-rábica. Levantamentos elementares dão conta de milhares de cães que perambulam pelas ruas, principalmente nos bairros populares das cidades. Explica-se, assim, o grande número de aplicações da vacina anti-rábica e, como conseqüência, de suas complicações neurológicas.

Dados preliminares, ainda sujeitos à confirmação, mostram uma incidência dessas complicações em proporção de $1: 2000$ pessoas vacinadas (com a vacina tipo Fermi modificada), nos últimos 5 anos. As formas mais leves são rever iveis espontâneamente, porém as mais severas podem levar ao óbito ou deixar seqüelas graves. Para tratamento destas complicaçōes foi considerada, desde 1961, a possibilidade da via intratecal para administração de corticosteróides em suspensão em veículos que retardem sua eliminação $1,2,8,10,11,13$.

A finalidade dêste trabalho é comparar os resultados obtidos com os tratamentos de repouso e sintomático e o baseado na administração do ACTH e/ou corticosteróides, com o acetato de metilprednisolona por via intratecal (IT).

\section{MATERIAL, E METODOS}

Foram analisados os resultados do tratamento de 33 pacientes, de ambos os sexos e idades diversas, que apresentaram complicações neurológicas, de extensāo e intensidade variáveis, em decorrência de aplicações da vacina anti-rábica, tipo Fermi modificada. Os pacientes foram internados no Hospital de Isolamento «Emilio Ribas» e divididos em três grupos para avaliação do tratamento: a) tratamento de suporte e sintomático (Quadro 1); b) tratamento igual ao grupo a acrescido de ACTH por via intravenosa (IV) nas doses de 25 a $50 \mathrm{mg}$ em infusão gôta-a-gôta ou por via intramuscular (IM) nas doses de 25 a $50 \mathrm{mg} / \mathrm{dia}$ e/ou corticosteróides pelas vias oral (VO), IM ou IV em doses variáveis de acôrdo com o caso e a via de administraçāo utilizada (Quadro 2); $c$ ) tratamento igual ao grupo

Trabalho apresentado ao IIr Congresso Brasileiro de Neurologia (Recife, PE 14 a 18 de julho de 1968): * Médico assistente do Hospital de Isolamento «Emilio Ribas» e Hospital das Clínicas da Fac. Med. Univ. São Paulo; ** Docente-Livre de Neurologia da Fac. Med. Univ. São Paulo. 
$b$ acrescido de acetato de metilprednisolona * por via IT, $4 \mathrm{ml}$ (160 $\mathrm{mg}$ ) injetados por punção raqueana $\left(L_{3}-L_{4}\right)$, após colheita do liquido cefalorraqueano para exame; a injeção foi feita de modo lento, com aspiracão freqüente (barbotage) para facilitar a dispersão do medicamento, sendo repetida cada 4 a 7 dias, conforme a resposta (Quadro 3).

\section{RESULTADOS}

Os resultados podem ser avaliados pela observação dos quadros 1, 2 e 3 . Note-se que nos casos do grupo a (Quadro 1), sem tratamento específico, nāo ocorreu óbito algum, tendo 8 pacientes obtido recuperação integral. Apenas um (caso 9) ficou com sequiela (bexiga neurogênica), estando atualmente em tratamento e observação (metilprednisolona em suspensão por via IT). No grupo b (Quadro 2) ocorreram dois óbitos, uma seqüela considerada irreversivel (paraplegia crural sensitivo-motora flácida com nivel em $D_{9}$, há cinco anos), 6 casos melhorados e 6 outros considerados curados. No grupo $c$ (Quadro 3), ocorreu um óbito causado por acidentes no aparelho de respiraçăo artificial; 6 pacientes foram considerados curados e dois melhorados.

\section{COMENTARIOS}

Admitindo-se ser a complicação neurológica o resultado de uma reação bàsicamente imunalérgica em resposta à exposiçāo repetida a um antígeno (vacina), parece razoável o emprego de drogas com atividades anti-inflamatórias e imunosupressoras, como os hormônios glicocorticóides $3,4,7,9$. No Hospital de Isolamento "Emílio Ribas" êsses hormônios foram usados no tratamento das complicações neurológicas depois de suspensa a administração da vacina, uma vez que êles podem ter interferência na produção de anticorpos anti-rábicos, especialmente os corticóides ${ }^{5}$.

Considerando a relativa lentidão dos processos de recuperação e o possivel beneficio da corticóidoterapia, foi considerada útil sua aplicação no espaço subaracnóideo, permitindo açāo direta e imediata no sistema nervoso e reduzindo, por outro lado, os efeitos sistêmicos ${ }^{10}$. Isto foi obtido pelo emprêgo da suspensão de acetato de metilprednisolona que se mantém em niveis razoàvelmente altos no líquido cefalorraqueano durante longo período de tempo ${ }^{13}$, diferentemente dos corticosteróides solúveis, que entram ràpidamente em equilibrio com os niveis plasmáticos ${ }^{8}$.

As respostas clínicas, com o método adotado, foram surpreendentemente boas, mesmo quando já se supunha estar a afecçāo entrando em fase de cronicidade (casos 25 e 32 ), apesar do tratamento anterior pelo método clássico.

Não foram observados fenômenos irritativos ou tóxicos, locais ou gerais, imputáveis a êsse método terapêutico, o que confirma, de modo geral, as referências da literatura ${ }^{1,2,6,10,11}$. Por outro lado, em um caso em que foi

* Depo Medrol do Laboratório Upjohn. 


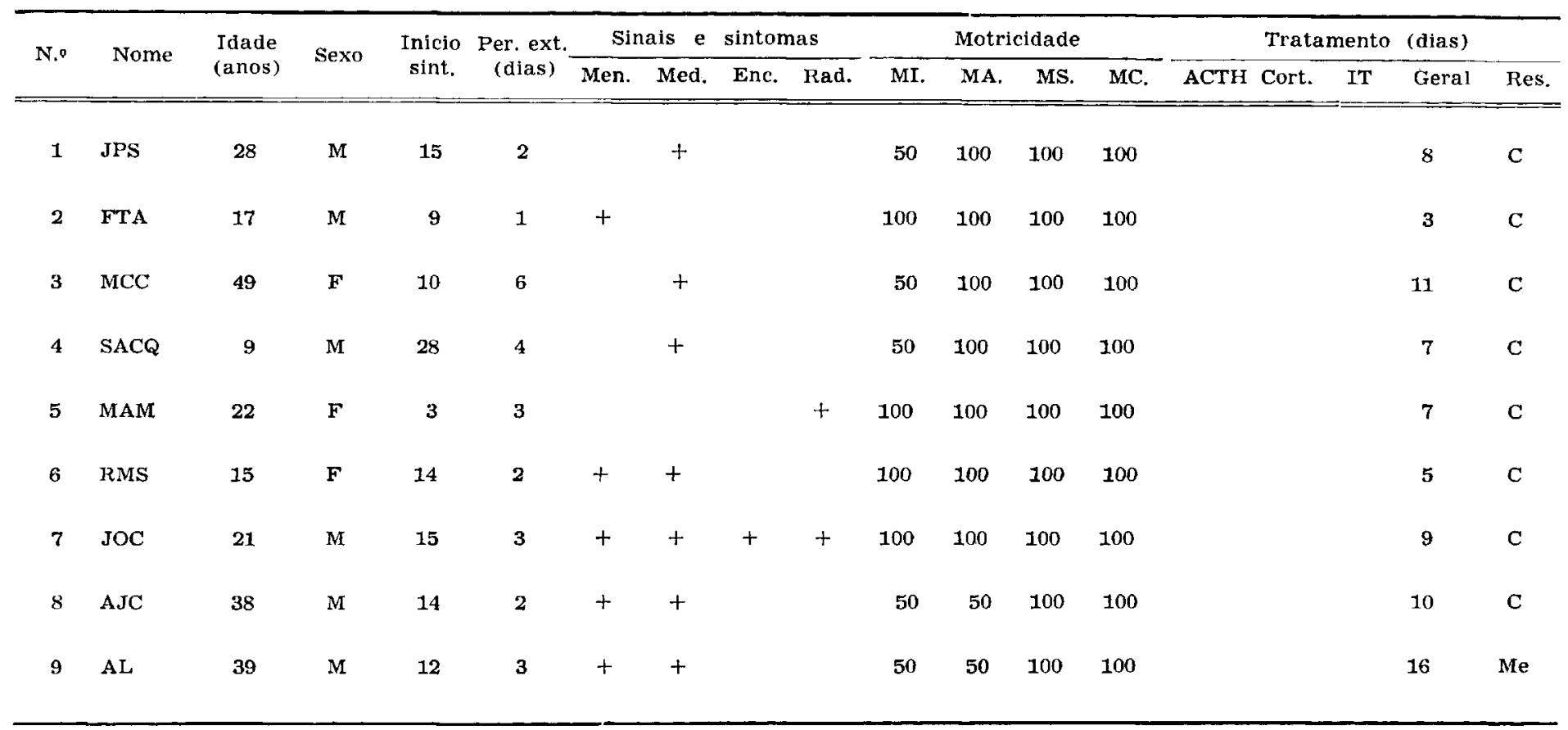

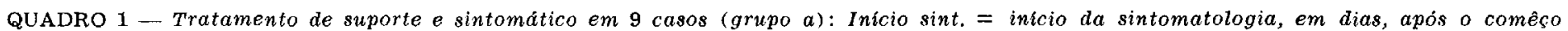

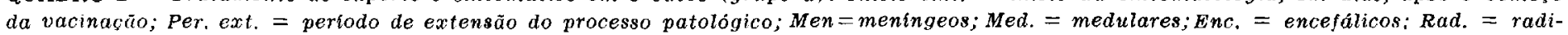
culares; $M I=$ músculos dos membros inferiores; $M A .=$ músculos abdominais; $M S .=$ músculos dos membros superiores; MC. $=$

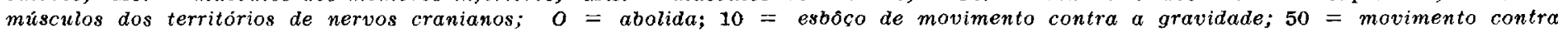

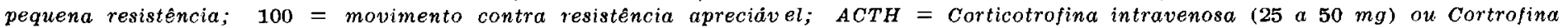

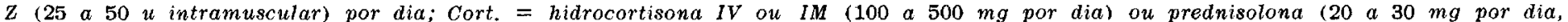

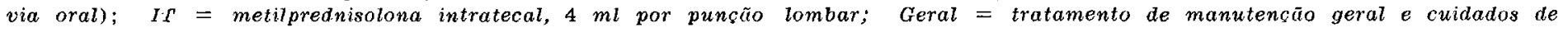
enfermagem; Res. = resultado do tratamento; $C=$ curado; $S=$ sequela; $O=$ obito; Me $=$ melhorado. 


\begin{tabular}{|c|c|c|c|c|c|c|c|c|c|c|c|c|c|c|c|c|c|c|}
\hline \multirow{2}{*}{ N.P } & \multirow{2}{*}{ Nome } & \multirow{2}{*}{$\begin{array}{c}\text { Idade } \\
\text { (anos) }\end{array}$} & \multirow{2}{*}{ Sexo } & \multirow{2}{*}{$\begin{array}{c}\text { Inicio } \\
\text { sint. }\end{array}$} & \multirow{2}{*}{$\begin{array}{l}\text { Per, ext. } \\
\text { (dias) }\end{array}$} & \multicolumn{2}{|c|}{ Sinais e } & \multicolumn{2}{|c|}{ sintomas } & \multicolumn{4}{|c|}{ Motrlcidade } & \multicolumn{5}{|c|}{ Tratamento (dias) } \\
\hline & & & & & & Men. & Med. & Enc. & Rad. & MI. & MA. & MS. & MC. & ACTH & Cort. & IT & Geral & Res. \\
\hline 10 & MF & 37 & $\mathbf{F}$ & 13 & 6 & + & + & & + & 10 & 50 & 50 & 100 & 6 & & & 20 & $\mathrm{C}$ \\
\hline 11 & MLF & 7 & $\mathbf{M}$ & 15 & 4 & + & + & + & + & 0 & 10 & 10 & 0 & 6 & 16 & & 16 & $\mathrm{O}$ \\
\hline 12 & MLA & 16 & $\mathbf{F}$ & 25 & 5 & + & + & + & + & 0 & 10 & 10 & 10 & 7 & 9 & & 9 & 0 \\
\hline 13 & EJ & 18 & $\mathbf{F}$ & 11 & 6 & + & $t$ & & + & 0 & 10 & 10 & 100 & 3 & $?$ & & $\mathbf{3}$ & $\mathbf{S}$ \\
\hline 14 & YA & 42 & $\mathbf{F}$ & 25 & 2 & + & & & + & 100 & 100 & 100 & 100 & 7 & 10 & & 10 & $\mathrm{C}$ \\
\hline 15 & GT & 29 & $\mathbf{M}$ & 11 & 2 & + & + & + & + & 0 & 50 & 50 & 50 & 6 & 8 & & 8 & Me \\
\hline 16 & JO & 15 & $\mathrm{~F}$ & 13 & 2 & + & + & & + & 0 & 50 & 100 & 100 & 16 & & & 58 & $\mathrm{C}$ \\
\hline 17 & NMSS & 15 & $\mathrm{~F}$ & 10 & 2 & + & + & & + & 0 & 50 & 100 & 100 & 7 & & & 10 & $\mathrm{Me}$ \\
\hline 18 & JSO & 52 & M & 12 & 3 & + & + & & + & 10 & 50 & 100 & 100 & 17 & & & 95 & $\mathrm{Me}$ \\
\hline 19 & $\mathrm{AL}$ & 25 & $\mathrm{M}$ & 15 & 2 & + & + & & + & 0 & 50 & 50 & 100 & 34 & 34 & & 79 & Me \\
\hline 20 & $\mathrm{CHS}$ & 24 & $\mathbf{M}$ & 9 & 4 & + & + & & + & 50 & 100 & 100 & 100 & 7 & & & 10 & C \\
\hline 21 & LAJ & 29 & M & 15 & 5 & + & + & & + & 50 & 50 & 100 & 100 & 22 & 22 & & 16 & $\mathrm{Me}$ \\
\hline 22 & AMA & 24 & $\mathrm{M}$ & 7 & 5 & + & + & & + & 50 & 50 & 50 & 100 & 21 & & & 20 & $\mathrm{Me}$ \\
\hline 23 & MOR & 11 & $\mathbf{F}$ & 17 & 4 & + & + & & + & 50 & 50 & 100 & 100 & 6 & & & 11 & $\mathbf{C}$ \\
\hline 24 & AMS & 38 & $\mathbf{M}$ & 12 & 7 & + & + & & & 50 & 100 & 100 & 100 & & 4 & & 4 & $\mathrm{C}$ \\
\hline
\end{tabular}

QUADRO 2 - Tratamento com corticotrofina elou corticóides acrescido de tratamento geral em 15 casos (grupo b). Mesma legenda que no quadro 1.

\begin{tabular}{|c|c|c|c|c|c|c|c|c|c|c|c|c|c|c|c|c|c|c|}
\hline \multirow{2}{*}{$\mathrm{N}, 9$} & \multirow{2}{*}{ Nome } & \multirow{2}{*}{$\begin{array}{c}\text { Iáade } \\
\text { (anos) }\end{array}$} & \multirow{2}{*}{ Sexo } & \multirow{2}{*}{$\begin{array}{c}\text { Inicio } \\
\text { sint. }\end{array}$} & \multirow{2}{*}{$\begin{array}{c}\text { Per, ext. } \\
\text { (dias) }\end{array}$} & \multicolumn{4}{|c|}{ Sinais e sintomas } & \multicolumn{4}{|c|}{ Motricidade } & \multicolumn{5}{|c|}{ Tratamento (dias) } \\
\hline & & & & & & Men. & Med. & Enc: & Rad. & MI. & MA. & MS. & MC. & $\widehat{\mathrm{ACTH}}$ & Cort. & IT & Geral & Res. \\
\hline 25 & MFS & 44 & $F$ & 21 & 7 & + & $\div$ & & + & 10 & 10 & 50 & 100 & 30 & 60 & 3 & 78 & $\mathrm{Me}$ \\
\hline 26 & GBO & 18 & $\mathrm{M}$ & 10 & 6 & + & + & + & + & 0 & 0 & 0 & 0 & & 1 & 1 & 2 & $O^{*}$ \\
\hline 27 & $\mathrm{ABH}$ & 23 & $\mathbf{M}$ & 12 & 5 & + & + & & + & 0 & 50 & 50 & 100 & & & 1 & 21 & $\mathrm{C}$ \\
\hline 28 & $\mathrm{BK}$ & 17 & $\mathrm{M}$ & 11 & 2 & + & + & & + & 0 & 25 & 50 & 100 & & & 2 & 26 & C \\
\hline 29 & MMN & 32 & $F$ & 14 & 4 & + & + & & + & 0 & 25 & 50 & 100 & & & 2 & 18 & C \\
\hline 30 & ENA & 21 & $F$ & 9 & 2 & + & + & & + & 50 & 50 & 100 & 100 & & & 1 & 7 & $C$ \\
\hline 31 & MU & 30 & $\mathbf{M}$ & 12 & 9 & + & + & + & + & 0 & 0 & 0 & 25 & 4 & & 2 & 53 & $\mathrm{C}$ \\
\hline 32 & PB & 25 & $\mathrm{M}$ & \pm 12 & 5 & + & + & & + & 0 & 50 & 100 & 100 & 50 & & 3 & 126 & $\mathrm{Me}$ \\
\hline 33 & AsW & 29 & $\mathbf{M}$ & 12 & 2 & + & + & & + & 0 & 0 & 50 & 50 & & & 8 & 30 & $\mathrm{C}$ \\
\hline
\end{tabular}

QUADRO 3 - Tratamento pela aplicacão da Metilprednisolona por punça lombar em 9 casos (grupo c). Mesma legenda que no quadro $1 . \quad$ * Acidente no aparêlho de respiração artificial. 
feita necropsia (caso 26), não foram encontrados fenômenos inflamatórios devidos à droga. Igualmente, não parece ter havido atividade sistêmica do hormônio. Estes fatos, que sugeriram a outros autores ${ }^{1,2,6,10,11,12}$ o emprêgo do mesmo método de tratamento em outras moléstias do sistema nervoso, nos autorizaram novas tentativas terapêuticas em algumas afecçōes neurológicas em que, até hoje, têm sido usados sòmente os métodos clássicos.

Considerando, finalmente, que a moléstia em foco tem caráter reversível espontâneamente, pelo menos de modo parcial, uma análise estatística demandará número muito maior de observaçōes, embora pareça justificada, nesta casuística, a superioridade do método intratecal empregado.

\section{R E S U M O}

Os autores estudaram 33 pacientes com complicações neurológicas da vacinação anti-rábica (tipo Fermi modificada) dividindo-os, quanto ao tratamento, em três grupos: a) tratamento sintomático e de manutenção ( 9 pacientes); b) tratamento igual ao do grupo $a$ acrescido de ACTH e/ou corticosteróides pelas vias oral, muscular ou venosa (15 pacientes); c) tratamento igual ao do grupo $a$ acrescido da administração de acetato de metilprednosolona em suspensão por via intratecal (9 pacientes).

Referem os autores um caso com seqüela no grupo $a$; dois óbitos, um caso com seqüela, 6 melhorados e 6 considerados curados no grupo $b$; um óbito (acidente com o respirador) e nenhuma outra complicação ou seqüela com os pacientes do grupo $c$ no qual 7 foram considerados curados e um melhorado. A impressão clínica, justificada pela recuperação mais rápida e completa, foi a de que os melhores resultados foram obtidos no grupo $c$.

S U M M A R Y

\section{Treatment of neurological complications in anti-rabies vaccination}

Neurological complications in 33 patients following antirabies vaccination (modified Fermi vaccine) were studied. For analysing purposes of the therapeutic effects the patients were divided in three groups: the first group with symptomatic treatment only ( 9 patients); the second with symptomatic treatment plus intravenous or intramuscular ACTH or corticosteroids (15 patients); the third with symptomatic treatment plus intrathecal methylprednisolone acetate ( 9 patients).

In the first group one of the patients remained with a neurological sequel. In the second group one patient also had a neurological sequel, two died, six improved and six had complete recovery. In the third group a patient died from a respirator accident, one improved and seven had complete recovery. The authors had the clinical impression that the results were considerably better in the third group of cases. 


\section{REFERENCIAS}

1. BOINES, G. J. - a) Remissions in multiple sclerosis following intrathecal methylprednisolone acetate. Delaware St. med. J. 33:230-235, 1961. b) Predictable remissions in multiple sclerosis. Delaware St. med. J.35:200-202, 1963. c) Erratic persistence of intrathecally injected methylpredinosolone acetate. Delaware St. med. J. 36:210-212, 1964.

2. BAKER, A. C. - Intrathecal methyilprednisolone for multiple sclerosis: evaluation by a standard neurological rating. Ann. Allergy 25:665-672, 1967.

3. BRANDRIN, M. W.; SMITH, J. W. \& FRIEDMAN, R. M. - Supression of experimental allergic encephalomyelitis by antimetabolites. Ann. N. X. Acad. Sci. 122:356-368, 1965.

4. BRIGGS, G. W. \& BROWN, M. W. - Neurological complications of antirabies vaccine; treatment with corticosteroids. J. Amer. med. Ass. 173:802-804, 1960.

5. BURNS, K. F.; SHELTON, D. F.; LUKEMAN, J. M. \& GROGAN, E. W. Cortisone and $\mathrm{ACTH}$ impairment of response to rabies vaccine. Publ, Hith Rep. (Washington) 75:441-445, 1960.

6. BUSKIRK, C. Van; POFFENBARGER, L. A.; CAPRILES, L. F. \& IDEA, B. V. - Treatment of multiple sclerosis with intrathecal steroids. Neurology (Minneapolis) 14:595-597, 1964.

7. FERRARO, A. \& ROIZIN, L. - a) Experimental allergic encephalomyelitis during and following cortone acetate treatment. J. Neuropath. 12:373-386, 1953. b) Hyperergic encephalomyelitis following exanthematic diseases, infectious diseases and vaccination. J. Neuropath. 16:423-445, 1957.

8. FISHMAN, R. A. \& CHRISTY, N, P. - Rate of adrenal cortical steroids following intrathecal injection. Neurology (Minneapolis) 15:1-6, 1965.

9. GAMMON, G. A. \& DILWORTH, M. J. - Effect of corticotropin on paralysis of experimental allergic encephalomyelitis. Arch. Neurol. Psychiat. (Chicago) $69: 649,1953$.

10. GOLdstein, N. P.; McKenZIE, B. F. \& McGUCKIN, W. F. - Changes in cerebrospinal fluid of patients with multiple selerosis after treatment with intrathecal methylprednisolone acetate. Proc. Mayo Clin., 37:657-668, 1962.

11. KULICK, S. A. - The clinical use of intrathecal methylprednisolone acetate following lumbar puncture. J. Mt. Sinai Hosp. 32:75-78, 1965.

12. SCHMIDT, E. - Erfahrungen mit der intrathecalen Corticosteroidbehandlung der Polysklerose und anderer neurologischer Erkrankungen. Psychiat. Neurol. u. med. Psychol. (Leipzig) 19:152-156, 1967.

13. SEHGAL, A. D. - Laboratory studies after intrathecal corticosteroids; determination of corticosteroids in plasma and cerebrospinal fluid. Arch. Neurol. Psychiat. (Chicago) 9:64-68, 1963. 